\title{
Process modelling issues in the design of a continuous flow process for the production of ibuprofen
}

\author{
Antonio Tripodi ${ }^{1}$, Rocco Martinazzo ${ }^{1}$, Gianguido Ramis ${ }^{2}$, Ilenia Rossetti ${ }^{1, *}$ \\ ${ }^{1}$ Chemical Plants and Industrial Chemistry Group, Dip. Chimica, Università degli Studi di Milano, \\ CNR-ISTM and INSTM Unit Milano-Università, via C. Golgi 19, 20133 Milano, Italy \\ ${ }^{2}$ DICCA, Università degli Studi di Genova and INSTM Unit-Genova, via all'Opera Pia 15A, 16100 \\ Genoa, Italy
}

\begin{abstract}
The continuous flow production of active pharmaceutic ingredients is a spreading applicative research field. Process simulation tools can be an effective mean for in silico process design and optimisation, but care should be taken in their use. We here report a paradigmatic example, the synthesis of ibuprofen. A first point is the need to select the most appropriate thermodynamic models to account for the properties of the involved species. The rich databases now available to collect thermodynamic and transport properties are often insufficient because, unfortunately, unconventional reactants and complex molecules are usually part of the recipe or found as intermediates or products. Furthermore, in some reaction steps ionic properties may be needed rather than those of the neutral molecules. All these points need a careful optimisation of the methods for the estimation of the properties, with possible huge discrepancies of the results.

Another key aspect is that most literature covers in depth the continuous reaction in micro- or mesofluidic devices, with poor attempts to connect on the same continuous line the products separation
\end{abstract}

\footnotetext{
* Corresponding author: Prof. Dr. Ilenia Rossetti, fax +39-02-50314300; email ilenia.rossetti@unimi.it
} 
and purification steps. Also in this case, options are available to set the process as fully continuous, but again, the key issue is the correct selection of the thermodynamic package, relying on very complex cases where experimental equilibrium data are missing. Improper predictions can neglect important issues, such as the unpredicted precipitation of salts, not envisioned during the simulation, but observed experimentally. Pertinent experimental data are here supplied to fill this gap.

Keywords: Continuous flow manufacturing; Ibuprofen; Active pharmaceutical ingredient (API); Microreactors; Process simulation; Extraction.

\section{Introduction}

Pharmaceutical industries are among the companies with higher revenues nowadays and their competitiveness is going to increase steadily. Batch production processes are dominant in the pharmaceutical industry and have multiple advantages, including equipment flexibility, high-fidelity quality control and the ability to recall specific batches, which is preferable due to regulatory problems, where traceability is compulsory. However, batch processes suffer disadvantages such as limited heat transfer and poor scalability due to mixing problems, as well as low operational asset efficiency [1]. They are also very wasteful: the pharmaceutical industry, produces $25-100 \mathrm{~kg}$ of wastes for every kilogram of a complex molecule synthesized [2].

Due to these inefficiencies, the production of pharmaceutical products and intermediates is moving towards alternative technologies that give, at the same time, the same reliability and effectiveness of batch operation, with some improvements from the point of view of selectivity, safety or feasibility, depending on the cases.

One of these alternatives is the Continuous Pharmaceutical Manufacturing (CPM). It offers many advantages: lower costs, reduced wastes, decreased time-to-market for new drugs. Continuous flow 
reactors can deliver significantly higher yields and better performance according to green chemistry prescriptions.

In addition to this, better heating and mixing capability can be achieved and intermediates that are unstable can be rapidly transferred from reactors to other vessels for subsequent transformation or workup [3], increasing the manageability of dangerous processes.

Indeed, only a small amount of risky streams is worked per unit time and volume, that can be generated and used immediately, avoiding the accumulation of large quantities. At the same time, micromixers and microreactors typically allow very efficient heat exchange, limiting safety issues in case of very exothermic reactions.

All these advantages lead to huge improvements from the chemical-engineering point of view and from an economic perspective: continuous operations can reduce operating expenditure by at least $90 \%$ and capital ones by at least $50 \%$, though these figures depend on the scale [4].

If continuous flow manufacturing of fine chemicals and pharmaceuticals is gaining more and more importance industrially for the mentioned reasons [5,6], the approach for process development is still mainly phenomenological. Only a few attempts have been proposed to model the processes, also due to the objective complexity of the reactions and compounds involved.

Therefore, we have selected one of the few examples very well detailed in the literature, the synthesis of ibuprofen, to study the process in depth and to evidence which lacking information would be needed for a straightforward process design and optimization. Hopefully, this can be a guide for checking the same critical issues during the CM design for other APIs or fine chemical.

From the inspection of the data available, detailed reaction kinetics is often missing, preventing reactor modelling, sizing and cost evaluation. Furthermore, the product separation is as much important as the continuous flow synthesis, but its conversion to a continuous protocol is not accounted for in sufficient detail. Finally, the molecules taken into account are often very complex, undergo very specific transformations, whose thermodynamic description is mostly unavailable. This 
can lead to unreliable predictions of heat consumption and release and to misleading predictions of products separation due to missing equilibrium and transport data.

We here propose hints to improve the experimental and computational results available in the literature by:

- linking the reaction and purification stages in order to simulate the whole ibuprofen production process, from the raw materials to the final API recrystallization;

- accounting for the heat released in the acid-base mixings, which is often as important as the reactions enthalpies. This affects the selection of the heat release strategy and is the critical point for the choice between scale-up (i.e. step up from the micro- to the meso-scale) or numbering-up (i.e. multiply the microreactors number to achieve the desired productivity);

- identifying the impact of the present purification procedures on the material balances of the whole process and not only to account for the final API purity;

- developing the separation section defining a simulated process strictly based on laboratory evidence with experimental data here collected;

- identifying the steps where the lack of detailed thermodynamic data lead to unreliable simulation and proposing the consequent adjustment.

Indeed, previous works on the simulation of this reaction provide important details on the reactive part, without tackling in detail the continuous separation. By contrast, the latter step is here strictly based on experimental recipes. The validation of simulation results with experimental evidence is here provided, evidencing some unpredicted effects (i.e. solids separation) and suggesting appropriate thermodynamic methods to represent correctly the physical behavior of the mixtures. Furthermore, we provide suggestions on heat management and on continuous crystallization that are not found in the literature. Thus, even if the quantitative information provided is specifically referred to ibuprofen synthesis, the logic approach and methods can be extended virtually to any example of continuous flow synthesis of API. 


\section{General flowsheet design}

Details on Ibuprofen as example of continuous API manufacturing, together with the full reaction scheme considered in this work are reported in the Supplementary Information file.

The flowsheet presented in this work (Figure 1) is based, for the reactivity step, on the work of Bogdan et al. [7] as rescaled by Jolliffe et al. [8]. On the contrary, the separation and crystallization sections are simulated here ex novo from an experimental protocol taken from Bogdan et al. [7] and similar patented procedures [9].

The three reactions are performed in the respective reactor coils. Then the separation of methanol is achieved in a flash. The separation from the unreacted organics is accomplished keeping ibuprofen as potassium salt and using three extractors. Subsequently ibuprofen is neutralized and further purified in other three extractors (recovering it in the organic phase in neutral form) and finally crystallised.

The critical features of this process are:

- the use of triflic acid (Trifluoromethanesulfonic acid, TfOH). This simplifies the raw material supply, since it acts both as solvent and as catalyst, but its complete miscibility with water and methanol poses a serious problem to recycle it;

- the quench of undesired parasitic reactions and the dissipation of solvation heats. This point determines significant heating and cooling duties;

- the choice of $\mathrm{KOH}$ as base, instead of $\mathrm{NaOH}$, prevents the early precipitation of ibuprofen as sodium salt [10]. This strategy avoids the co-precipitation of $\mathrm{NaCl}$, which would contaminate the ibuprofen crystals [11]. $\mathrm{NaCl}$ precipitation is expected due to the large quantity of methanol with respect to water;

- methanol is not an effective anti-solvent for the potassium salt of ibuprofen [10]. Therefore, a rather complex purification procedure is needed downstream, with the switch of the API between the polar and the non-polar solvent and multiple washings of both phases; 
- downstream the last reactor, the first washing of the aqueous phase with the organic extraction solvent depends crucially on the methanol evaporation achieved in the previous flash step. Indeed, methanol splits between the water and most organic solvents suitable for the extraction. Methanol is relatively high boiling $\left(65^{\circ} \mathrm{C}\right)$ with respect to the thermal stability of ibuprofen [12], thus this step must be treated carefully when a continuous flash-like evaporation is designed to scale up the standard solvent evaporation technique under vacuum accomplished at lab scale. If, on the other hand, methanol is not removed prior the acidic work-up, the recovery of ibuprofen from the aqueous phase into the organic one becomes totally different due to the high ibuprofen solubility in the alcohol.

Since the API has to be separated from both organic (e.g. unreacted isobutylbenzene) and polar (e.g. acetic acid) residues, the adopted process design (yielding the soluble ibuprofen potassium salt in

presence of methanol) imposes two purification steps in series. The solvent may be the same [7], but at least a couple of critical points can be foreseen, namely:

- the $\mathrm{HCl}$ addition into a high $\mathrm{pH}$ solution introduces a severe heat-release, together with an additional separation device needed for the potassium chloride (scarcely soluble in methanol and thus oversaturated in the foreseen water flow). Otherwise, a careful reassessment of the downstream hydrodynamic is needed to cope with the solid salt through the separation line;

- the choice of an organic extractor different from the crystallization solvent [7] complicates the chemical balances[13,14].

\subsection{Reactive section}

All the details and considerations on the choice of the thermodynamic models are reported in the Supplementary Information file. 
Reaction 1: the Friedel-Craft acylation, involves IBB and propionic acid to form the first intermediate. The reaction is catalyzed by $\mathrm{TfOH}$ that is also used as a solvent. The reaction is operated at $150^{\circ} \mathrm{C}$ and 1 bar.

The reaction heats were calculated using the same $\Delta_{\mathrm{f}} \mathrm{H}^{0}$ values derived by Jolliffe et al. with the Gani method (see [8] and references therein) for ibuprofen and its precursors. In principle, this is not fully correct, since in [8] the reference state is the liquid phase, while Aspen Plus always considers the ideal gas state. Therefore, in the first reaction heat there is a sensible difference $(+95 \mathrm{~kJ} / \mathrm{mol}$ instead of $+143 \mathrm{~kJ} / \mathrm{mol}$ ) due to the different specific heat and the condensation heat of the first ibuprofen precursor, which unfortunately could not be adjusted using experimental data.

Reaction 2: the stream exiting the first reactor is cooled to $0^{\circ} \mathrm{C}$. TMOF and $\mathrm{PhI}(\mathrm{OAc})_{2}$ are dissolved in $\mathrm{MeOH}$ and their temperature is set to $0^{\circ} \mathrm{C}$. The two streams are sent to the second reactor where a 1,2-aryl migration, mediated by $\mathrm{PhI}(\mathrm{OAc})_{2}$, occurs at $50^{\circ} \mathrm{C}$ to form the second intermediate with $\mathrm{TfOH}$, used again as acid catalyst.

Also in this case, the calculated reaction heat differs for the already mentioned reasons. While the passage from the first to the second API precursor should not introduce a great error (the difference of $\Delta_{\mathrm{f}} \mathrm{H}^{0}$ is the same as in the literature and the handling of the specific heats by Aspen Plus should be the same), the estimate made on the $\mathrm{PhI}(\mathrm{OAc})_{2}$ molecule may be biased in our calculation as well as in the referenced one [8], given also the lack of laboratory data for this compound.

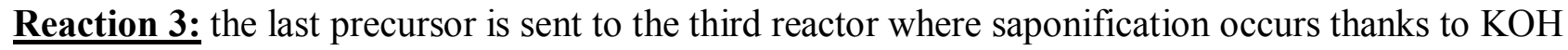
that was previously dissolved in a $\mathrm{H}_{2} \mathrm{O} / \mathrm{MeOH}$ mixture in a separated stream. The product of this reaction is the potassium salt of ibuprofen.

The kinetic parameters for this reaction have been derived from the computational estimation by Jolliffe et al. [8], with the needed unit conversion and several modifications. While the cited authors provide a stoichiometry based on $\mathrm{KOH}$ as reactant, we preferred to use water in order to obtain 
ibuprofen, as a product, instead of the ibuprofen salt. This choice was determined by the severe difficulties experienced trying to define the thermodynamic parameters of general electrolytes: while this work was mandatory for the triflic acid due to its quantitative importance, the relatively low amount of ibuprofen and its weak dissociation, allows to neglect its anion. Accordingly, to maintain a pseudo first-order kinetic, the given reaction rate was rescaled for the water concentration, and finally we switched the independent integration variable from time to reactor volume. Indeed, the Aspen Plus ${ }^{\circledR}$ engine allows electrolytes only in equilibrium reactions, not in kinetic ones. So, as a first step, we took the kinetic constant provided in the literature at the given $\mathrm{KOH}$ concentration, and rescaled it for the water volume normalized to the reactor volume. Then we dropped any orderdependence on water, reducing to a pseudo-first order reaction. Actually, due to this simplification, the $\mathrm{KOH}$ role is hidden in the kinetic constant rescaling.

This caused of course a mismatch in the duty calculation with the cited reference [8], since the reaction $\Delta_{\mathrm{r}} \mathrm{H}$ changed. The consistency of our approach is based on keeping the same $\Delta_{\mathrm{f}} \mathrm{H}^{\circ}$ for ibuprofen and its precursor, while the error within the reactor is of the same order as [8].

\subsection{Acid-base reactions}

The electrolytic nature of the employed streams imposes a review of the heat calculations taking into account the acid-base chemistry. The conditioning of the second reactor feed stream to $0{ }^{\circ} \mathrm{C}$ depends on the solvation of the triflic acid within the methanol (being the reactions endothermic), as also observed experimentally [7]. A set of equilibrium reactions was then defined, whose parameters were fully retrieved in the AP databases: water autoprotolysis, $\mathrm{AcOH}, \mathrm{HCl}$ and $\mathrm{KOH}$ dissociations. The propionic acid and triflic acid dissociations had to be defined ex novo, so they were included into a separate 'Chemistry ID' in order to be used only when strictly needed and not overload the calculation of the blocks and the streams flash. 
The acid-base heat release starts actually within the first reactor, as the water formed as a reaction product is protonated by the $\mathrm{TfOH}$ present in large excess.

For this, we added an external 'Calculator' block that records the formed water and assigns proportionally an additional heat of $+79 \mathrm{~kJ} / \mathrm{mol}$ (for the $\mathrm{TfOH}+\mathrm{H}_{2} \mathrm{O} \rightarrow \mathrm{TfO}^{-}+\mathrm{H}_{3} \mathrm{O}^{+}$reaction [17]) to the reactor duty. Indeed, acid-base reactions expect water as a solvent, which is initially not present, $\mathrm{TfOH}$ being both the solvent and acid. The same approach is followed to calculate the heat in the mixer at the second reactor inlet, because the methanol protonation $\mathrm{TfOH}+\mathrm{MeOH} \rightarrow \mathrm{TfO}^{-}+$ $\mathrm{MeOH}_{2}{ }^{+}(-70 \mathrm{~kJ} / \mathrm{mol}[17])$ cannot be specified in the 'Equilibrium Chemistry' AP framework due to the lack of thermodynamic data for the $\mathrm{TfO}^{-}$and $\mathrm{MeOH}_{2}{ }^{+}$species. In this way, we were able to include in the simulation the correct assessment of the heat duties as consistent improvement with respect to available literature.

As the triflic acid comes in contact with a large quantity of water at high $\mathrm{pH}$ in the mixer before the third reactor, the standard electrolytic framework of AP can be used and the block TR1HX3 can now employ automatically the full acid-base chemistry (being water present also at its inlet): since the AP routines now refers to the still unknown $\Delta_{\mathrm{f}(\mathrm{liq})} \mathrm{H}^{0}$ and $\Delta_{\mathrm{f}(\mathrm{liq})} \mathrm{S}^{0}$ values for the $\mathrm{TfO}^{-}$species, these quantities were adjusted to match the reported solvation heat of this acid in water in a parallel test block. Notice that the mass balances of the system are substantially correct, because water is a stronger base than methanol, so the $\mathrm{MeOH}_{2}{ }^{+}$cations present to this point are turned into $\mathrm{H}_{3} \mathrm{O}^{+}$ions, correctly quantified. The pKa value of the triflic acid at $25{ }^{\circ} \mathrm{C}$ was set to -12.5 after reviewing literature data $[18,19]$.

\section{Extraction and separation sections}

\subsection{Preliminary assessment of ibuprofen recovery}


The mixture exiting the third reactor contains methanol, water, organic residuals and the ibuprofen and triflate potassium salts. To simulate this section we chose the experimental procedure proposed by Bogdan et al. [7], rather than to reproduce the same calculations of Joliffe et al. [8,13], to improve the recovery of the API. The procedure was as follows:

a) first methanol extraction with an additional water dilution: these passages grant an easier split between the apolar and the polar phases;

b) an organic solvent is used to extract the unreacted aromatic molecules and esters;

c) the solution $\mathrm{pH}$ is decreased with $\mathrm{HCl}$ to turn ibuprofen into its neutral form;

d) fresh organic solvent is added to remove neutral ibuprofen from the aqueous solution of salts and triflic acid, while additional water is added to neutralize the solution $\mathrm{pH}$.

The computation of these steps, however, is hard due to the very high ionic strength of the aqueous phase. Furthermore, such phase cannot be modelled by the UNIFAC package and we did not retrieve ibuprofen solubility data for solvents other than pure water. It is possible to assume that the solvated ions decrease water solubility in the organic phase, but methanol could undergo a "salting-out" effect [20-22]. Moreover, also the fact that ibuprofen potassium salts are soluble in methanol [10] has to be considered. For these reasons, we decided to treat this stage without considering the ions, but allowing a split fraction for the ibuprofen into the solvent as high as that in methanol. We also assumed that the other organics were instead dissolved completely in the organic phase. Since the laboratory protocols reported in the literature suggest three washings, our model is based on three subsequent separators fed with fresh solvent.

The Pfizer list [23] of solvents, especially for pharmaceutical applications, was used as starting reference. For any solvent with toxicity or environmental issues the list also proposes greener alternatives. Six solvents (toluene, hexane, heptane, dichloromethane, isopropyl acetate and $m$ xylene) were here tested for extraction, based on their ternary diagrams with water and methanol (Fig. S3 and S4). Toluene and hexane [8], besides diethyl ether [7] were already proposed before for this separation. Heptane was chosen here as a replacement for hexane, which is more toxic, also on the 
ground of laboratory experiments [11]. Dichloromethane is the recommended alternative to other chlorinated solvents, such as chloroform, dichloroethane or carbon tetrachloride [23]. Isopropyl acetate is a good alternative for acetonitrile, another commonly used extraction solvent and has very similar characteristics. m-xylene has been considered because it is very similar to toluene, but less volatile. The hazards of xylene are well documented, but most of the less-expensive alternatives to xylene do not have the same miscibility with alcohols. However, as it is relatively low boiling, it can be easily separated and recovered [24].

\subsection{Experimental validation of calculated extractions}

Due to the fact that the extraction and, consequently, the separation and crystallization of ibuprofen were not described quantitatively in the literature, we supported this part also experimentally, analyzing by NMR the composition of the aqueous and organic phases separated after extraction. Experimental details are also reported in the Supporting Information.

As already said, Aspen Plus ${ }^{\circledR}$ does not provide a thermodynamic model fully suitable for the extraction section, due to the co-presence of electrolytes $\left(\mathrm{H}^{+}, \mathrm{Cl}^{-}, \mathrm{K}^{+}, \mathrm{OH}^{-}\right)$and solvents, respectively well represented by the ENRTL and UNIFAC thermodynamic packages. Using UNIFAC to compute a realistic extraction introduces errors since the ionic force of the solution is not properly taken into account and this may result in different miscibility or solubility with respect to non-electrolytic systems. Therefore, we performed several extraction experiments in order to validate the AP calculations on the recovery of the ibuprofen.

We considered the re-acidified mixture containing ibuprofen in its neutral form in a mixed methanolwater solvent, which should represent the less favorable case due to the significant affinity of this alcohol for the polar phase. Thus, this estimation of ibuprofen recovery is the most conservative. 


\section{Simulation results}

\subsection{Reactive part}

$\underline{\text { Reaction 1: }}$ this calculation foresees a reactor duty of $251 \mathrm{~J}$ per gram (2.9 $\mathrm{W}$ at the flowrate of 41.6 $\mathrm{g} / \mathrm{h}$, instead of the $3.36 \mathrm{~W}$ reported in the literature), to heat the reagents from 25 to $150{ }^{\circ} \mathrm{C}$ and to supply the reaction enthalpy. The heat released by the protonation of the formed water is $c a .536 \mathrm{~J} / \mathrm{g}$ of isobutylbenzene $(0.87 \mathrm{~W}$ in total $)$ which means a $30 \%$ decrease in the actual reactor duty with respect to data reported elsewhere [8].

Reaction 2: being the reaction less endothermic than the others (and being the mole fraction of the reactant $2 \%$, only), the computed power is needed essentially to heat-up the mixture from $0{ }^{\circ} \mathrm{C}$ to 50 ${ }^{\circ} \mathrm{C}$. Our calculations foresee a total of $4.2 \mathrm{~W}$ for the $166 \mathrm{~g} / \mathrm{h}$ treated. The difference with literature duty (3.04 W [8]) was mainly attributed to the treatment of $\mathrm{KOH}$ as solvated ions, with a different effect on the mixture heat capacity than those predictable with non-electrolytic models. Anyway, much of this heat input would be compensated by the heat released when the triflic acid exiting the first reactor comes into contact with the methanol that carries the second reagents group, which is as high as $4.0 \mathrm{~W}$. If the mixer would not be ice-bathed, this heat would rise the temperature of the mixture to $c a .62{ }^{\circ} \mathrm{C}$. Though AP finds the mixture still within its boiling point, this value (coupled to the already discussed approximation) is consistent with the potential off-gasing issues mentioned in experimental works [7], also considering that the methanol mole fraction is $70 \%$ (even higher than the $50 \%$ at the third reactor exit). $12{ }^{\circ} \mathrm{C}$ difference with respect to the desired reaction temperature would then leave only $0.2 \mathrm{~W}$ to the duty to be granted.

According to these estimations, the reagents cool-down to $0{ }^{\circ} \mathrm{C}$ is not strictly needed, and comes probably from the fact that this temperature is more easily obtained, with laboratory materials and practice, than other values. In these conditions, the reactor heat input is mainly due as power to heat 
the reagents. If this is $4.0 \times 50 / 62=3.2 \mathrm{~W}$ (from 0 to $50{ }^{\circ} \mathrm{C}$, if the mixer is ice-cooled), while $1.0 \mathrm{~W}$ is due as reaction heat (our calculation), then the ratio between the powers is of 3:1. A possible scaleup to larger sizes would then have to consider at least two zones with markedly different thermal profiles (and stresses) and heat loads.

With a more careful thermal management, the overall heat duty of this process stage could be simplified, leading ideally to a single cooling stage keeping the reagents stream at $50{ }^{\circ} \mathrm{C}$ (our calculations foresee globally $3.6 \mathrm{~W}$ for the ice-bath cooling, before another $0.8 \mathrm{~W}$ contribution to compensate the acid-base enthalpy) before a mildly heated-up reactive section ( $1 \mathrm{~W} c a$.). The calculation of the acid chemistry switches the problem of the heat exchange from a heat-input to a heat-release, but lets also surmise that less drastic thermal profiles would be feasible.

Reaction 3: also in this case, the differences in the calculation of reaction enthalpies and mixture specific heat lead us to a global duty $(3.2 \mathrm{~W}$, i.e. $1500 \mathrm{~W} / \mathrm{g}$ of ibuprofen $)$, significantly different from the already reported one $(4.85 \mathrm{~W})$. Nevertheless, we can account for a heat of $7.4 \mathrm{~W}$ coming from the neutralization of the triflic acid with the added $\mathrm{KOH}$ (almost double of the higher reactor duty, to maintain the mixture within $51{ }^{\circ} \mathrm{C}$ ). The excellent heat transfer properties of the reaction coil, addressed by Bogdan et al. [7] to explain their safe mixing of reactants, is then confirmed quantitatively. However, it remains evident that also in this case almost half of exchange surface is devoted to cooling issues before the reaction starts. We have to point out that the ENRTL model calculates a boiling point of $56-57^{\circ} \mathrm{C}$ for this mixture, below the required reaction temperature of 65 ${ }^{\circ} \mathrm{C}$, so we increased this stage pressure to $1.5 \mathrm{~atm}$ to ensure a liquid phase only. Notice also that pressurized conditions are already foreseen in the similar route of Snead et al. [25], it is then possible that the experiment of Bogdan et al. [7] was not accomplished under fully optimized pressure conditions. 
The highest uncertainty in the description of this part should lie in the solvation enthalpy of TfO-' but, due to the large fractions of well known chemicals (water and methanol for a total of $63 \% \mathrm{~mol} / \mathrm{mol}$ ), we deem that it is not very important.

\subsection{Water-organic phase split}

A first comparison between the UNIFAC and UNIQUAC models shows that the former is more robust when describing the separation between water and increasingly longer alkane molecules in presence of methanol (Fig. S3, Supplementary Information), while for other molecules (Fig. S5, Supplementary Information) the two models are in better agreement with each other. Thus, ternary diagrams with water and methanol were calculated for each solvent according to UNIFAC. The results are reported in Fig. S4 (Supplementary Information).

All the selected solvents were practically immiscible with water, except isopropylacetate, but miscibility with methanol was very different. This influenced the split ratio of ibuprofen and the minimum flowrate of the extracting solvent to be used.

Some discrepancies with literature [8] were observed for toluene, which reported a single phase between 25 and $65^{\circ} \mathrm{C}$ for the lower solvent quantities, whereas in the present simulation we obtained two different phases for any solvent flowrate.

To check the effect of temperature, hexane was exemplified as extraction solvent: a variation from 10 to $35^{\circ} \mathrm{C}$ (Fig. S6 and S7) did not affect the trend of the organic phase split fraction, so we selected $25^{\circ} \mathrm{C}$ as preferable extraction temperature. An inverse trend with respect to what is expected (i.e. more extractor $=$ less recovery) was detected at $35{ }^{\circ} \mathrm{C}$ for the minimum solvent quantity, outside the range chosen for our further calculations (for an aqueous inlet of $9.5 \mathrm{~g} / \mathrm{h}$ of the API, $139 \mathrm{~g} / \mathrm{h}$ of methanol and $35.5 \mathrm{~g} / \mathrm{h}$ of water).

Finally, for all the tested solvents, except toluene and m-xylene that for very low amounts (up to 30 $\mathrm{g} / \mathrm{h}$ for m-xylene and up to $40 \mathrm{~g} / \mathrm{h}$ for toluene) did not trigger the phase-split, the amount of ibuprofen 
in the organic phase increased with increasing the extraction solvent flowrate (Fig. S8). The denser phase (bottom) was always the aqueous one except for the case of dichloromethane.

Isopropyl acetate, hexane and heptane required very low solvent flowrates for splitting, but with the former, the loss of ibuprofen in the lighter organic phase was much higher. Hexane and Heptane confirmed the better choices from this point of view.

Another parameter to control was the loss of methanol in the organic phase, which can give a reasonable idea of what the API salt losses would be. It can be noticed (Fig. S9) that isopropylacetate is, as expected, unsuitable due to its high miscibility with methanol. The best options from this point of view are hexane, heptane, toluene and m-xylene. Alkanes were selected, instead of aromatics, based on a comparison with experimental works, also because they can be used as crystallizing solvents. Heptane was then preferred to hexane as it is easier to handle, for the reasons mentioned above (Fig. S10 and S11).

Experimental validation tests are reported in the Supporting Information file (ibuprofen solubility and phase separation).

\subsection{Calculations for the separation section}

The first calculation presented (Fig. S10) is a benchmark of the UNIFAC model against experimental ternary data for the water-methanol-heptane mixture, as reviewed in [26]. We can see that the agreement was good, especially towards the region of interest $\left(\mathrm{MeOH} / \mathrm{H}_{2} \mathrm{O}=0.4 \mathrm{~mol} / \mathrm{mol}\right.$ for the aqueous phase).

The comparison of the laboratory test with UNIFAC calculations with different heptane quantities is shown in Fig. S11. For simplicity we excluded from the simulated mixture the residuals of acetic acid, propanoic acid and the relatively small quantity of TMOF. Overall, the calculated partition factor of ibuprofen (ratio of the organic/water mole fractions) is of the same order of magnitude of the experimental one. We noticed that the UNIFAC representation for the TfOH obtained via the 
built-in Bondi method is insufficient for a reliable estimation of its behavior, since this species is foreseen to split between the phases while its affinity for a polar solvent (which is also a conjugated base) is much higher than that for an alkane [19].

More important is the fair agreement obtained considering the acid as additional water, that means to fix its split fraction and postulate an ibuprofen solubility in this solvent as low as in water. When the solvent flowrate increases, the partition factor adjusts to lower values as expected, and this lets us also surmise an over-estimation of the ibuprofen recovery in the organic phase when the heptane amount is very low.

It is also interesting to notice the sensitivity of the simulation on the assumed methanol quantity. There was a great variation of the ibuprofen solubility in the polar phase as the water/methanol ratio varied. The solubility data for ibuprofen in methanol are relatively dispersed (Fig. S12). Other sparse data on the co-solvent system water-methanol as a function of temperature [27] agree with a systematic study at the fixed value of $25^{\circ} \mathrm{C}$ [28] only in the range of water fractions $0.1-0.8$. Outside these values, the solubility calculation given by Filippa et al. [28] underestimates the value for pure methanol [29] and overestimates that for pure water [30]. Anyway, taking $0.03 \mathrm{~mol} / \mathrm{mol}$ as the reference value for the ibuprofen solubility in heptane at $25^{\circ} \mathrm{C}[31]$ and comparing it with the fitting formula reported in [28], one can appreciate (Fig. S12) the sheer increase of the partition factor as the methanol fraction in the polar phase decreases, which fully explains the calculation sensitivity to the $\mathrm{TfOH}$ behavior and to apparently minor issues as considering or not the $\mathrm{OH}^{-} / \mathrm{H}_{3} \mathrm{O}^{+}$pairs as moles of water. The fact that higher solvent flows shift the UNIFAC model toward a better agreement both with the presented data and our lab tests, hints to another calculation issue when ibuprofen is in the super-saturated range, but no solid phase is yet modeled.

The methanol gross separation is represented through a single stage liquid-vapor equilibrium under mild vacuum conditions ( $0.8 \mathrm{~atm})$, to calculate a bubble point lower than the thermal stability limit of ibuprofen, and afterwards additional water is added to decrease the methanol fraction in the liquid. 
Since at this stage the API is still in its anionic form, this helps to keep it in the polar phase (ibuprofen potassium salt are soluble also in methanol [10]).

The UNIFAC-based analysis on the mixture exiting the methanol-separation flash unit is reported in Fig. S13, together with the selected working zone for each extraction stage. The correct calculation for triflic acid was fundamental to determine the outputs and the acid itself was $95 \%$ assigned to the polar phase. The heptane flow was set to a relatively high value, to compensate for a possible methanol underestimation, the ibuprofen and methanol split fraction to the water phase were conservatively set to $95 \%$ and $90 \%$, respectively.

A similar analysis for the final separation conditions (Fig. S14) has helped to quantify the total heptane amount as $1.6 \mathrm{~mol} / \mathrm{h}$. In this case, the flow is adjusted to optimize the ibuprofen split fraction. Additional water is added at each stage to buffer the $\mathrm{pH}$ [7], that rises from 0.3 in the acidified stream to the value of 5.9 in the last polar byproduct (the $\mathrm{pH}$ results given by AP for the organic phase are not reported, since the calculation refers the $\mathrm{OH}^{-}$residuals in the $0.13 \mathrm{~mol} \%$ water content). The calculated ibuprofen loss in these stages is negligible. From all the described results, it is shown that a substantial reduction of the methanol content of the mixture, as outlined by the experimental works $[7,11]$, is the way to achieve a better ibuprofen recovery in this two-steps separation layout, while the calculation made at high alcohol contents [13] may be unpredictably biased and too optimistic.

\subsection{Crystallization}

The specific heat of the ibuprofen and its heat of formation were treated as the pure (racemic) compound. This approach may lead to errors in the solvation enthalpy calculation, yet the relatively low solubility of ibuprofen in heptane should limit this issue (see also [32] for a similar approach). Following for simplicity the approach of a supersaturating-induced crystallization, rather than a seedinduced one [33], we started with a representation of the solubility within the 'Crystallizer' block after a review of available data (Fig. S15). 
The best fit was calculated from the same Figure S15 as $\ln (\mathrm{x})=18-6295 / \mathrm{T}$ on the basis of linear regression with least-square residuals, where $\mathrm{x}$ is the molar fraction and $\mathrm{T}$ is expressed in Kelvin.

To foresee the removal of excess heptane, we checked the effect of nitrogen partial pressure and total pressure (Fig. S16), finding that the former parameter was much more important in order to operate the solvent evaporation below $60-65{ }^{\circ} \mathrm{C}$. To achieve a good difference between the ibuprofen fraction in the evaporator liquid $(2 \% \mathrm{~mol} / \mathrm{mol})$ and a designed crystallization temperature not lower than $5{ }^{\circ} \mathrm{C}$, we choose to work with 2.5 moles of nitrogen per mole of heptane at $40^{\circ} \mathrm{C}$, to avoid a too steep increase of the heat duty.

The yield of ibuprofen after this last stage was $4.72 \mathrm{~g} / \mathrm{h}$. The overall ibuprofen mass balance throughout the separation section is summarized in Fig. 2, while details on the calculation of this block are reported in the Supporting Information.

The loss in the first separation section represents the prudential assumption that a part of the API salt could be transferred to the organic phase alongside the methanol, while the ideal behavior of the second section derives from the fact that there is not enough methanol to effectively drag the neutral ibuprofen in the polar residue. While the overall $61 \%$ separation yield is in line with the experimental data [7], we have to specify that the main loss of our calculation comes from the one-stage crystallization ( $24 \%$ of the produced API, starting from a recovery of $85 \%$ ) and derives from the relatively high heptane flow employed, while the cited experiments address higher losses to the second separation stage (the loss due to the crystallization is from $68 \%$ to $51 \%$, only the $17 \%$ ). This difference may be the result of the computational issues so far addressed, but it may also depend on the different water quantities used for the washing (besides the difference of a batch process from a continuous one, the cited papers do not report these details) and, moreover, on the different methanol residuals.

\section{Conclusions}


The study of a continuous flow process for the production of ibuprofen was carried out using Aspen Plus ${ }^{\circledR}$ as process simulation tool. This reaction was set as a paradigmatic example, given the different records present in literature, to discuss the importance of a correct choice of the thermodynamic descriptors for the different process stages. Using ENRTL as the overall model due to the polar or ionic nature of many of the reactant species while using UNIFAC to model the separation steps with the more common solvents present in high concentrations during the purification is a key insight. The handling of the enthalpies of reaction and mixing for the reaction stages with low water concentration is needed before the introduction of the water stream allowing AP to start automatically calculating the acid-base effects.

In addition, it is highly recommended to validate carefully each step against experimental data to avoid unpredictable effects (e.g. in this case salts precipitation) due to partial data availability. For instance solubility data in pure solvents are mostly available, while solvents mixtures may deeply influence this parameter. Using experimental single-stage separation data to support and validate the aspen calculation for the mixed solvent and electrolyte system is of great importance as well as the selection of conservative conditions for the prior methanol flash step.

The Elec-NRTL thermodynamic model was found adequate to describe the mass balances of the reaction section, while mixing and heat exchangers were modeled with the substantial help of literature data for the key compound $\mathrm{TfOH}$. Despite its importance in this process, this species is not already fully parametrized in the proprietary archives available. The presence of electrolytes, prevents a safe estimation of the properties of the mixture by the predictive UNIFAC method, that was instead used for the separation section, involving accurate description of liquid-liquid equilibria with a definitely non-polar solvent, which cannot be safely accounted for by using the NRTL model.

A preliminary study of the recovery of ibuprofen allowed the selection of the most appropriate extraction solvent that is also used for the crystallization stage. However, due to the very high 
complexity of the mixture, an experimental validation of the results was needed, together with a review of the pertinent literature.

The UNIFAC model satisfactorily represented the separation with several solvents and predicted correctly the best one for the extraction, but it could not represent as well the presence of electrolytes. The experimental tests evidenced the formation of co-products $(\mathrm{KCl})$, unpredicted during the simulation, that can give problems during the extraction due to the formation of a considerable amount of precipitate. This also imposes an unpredicted separation of the solid in the scaled-up flowsheet.

The NMR analysis showed that the amount of ibuprofen split in the aqueous and organic phases was different form the predictions of Aspen Plus ${ }^{\circledR}$, due to a very complex solvent mixture, but the results showed a sufficient level of agreement and, moreover, their difference can be fully explained by the highlighted criticisms of the selected reaction solvent, not to mention the non-negligible discrepancies that can still be found in the reviewed data.

According to this study, heptane can be considered as the best solvent for ibuprofen recovery and a convenient recrystallization medium. If this last stage is performed continuously, with a surnatant recycle, its recovery fraction approaches $100 \%$ at least in a preliminary design, confining the API losses to the washing stages.

Any eventual scale-up of the reactive section will have to consider acid-base solvation heats, as high as the endothermal duties of the microreactors, and even more critical for the equipment because they are developed under higher temperature gradients.

A flash section before the extraction is needed to reduce the methanol content and thus to substantially decrease the ibuprofen loss in the re-acidified stream undergoing the double separation layout. We can clearly identify the $3^{\text {rd }}$ reactor feed as the first critical point of the whole downstream section. Besides the cooling duty required by the mixing of a strong base with the triflic acid, a different choice of the hydroxide source would most likely determine a totally different separation design: ibuprofen sodium salts can be precipitated from the hydro-alcoholic stream, but neutral ibuprofen 
requires a $\mathrm{pH}$-buffering that leads to the contemporary precipitation of $\mathrm{NaCl}$. Solid settling was already reported to be a problem in microreactor channels.

The use of methanol as mixing and reaction solvent, with the related issue of its flash evaporation conditions, becomes the second design-determining step, because it affects the salt solubility (whatever the base employed) and at the same time the relative solubility of neutral ibuprofen between the polar and the organic solvents.

Also the non-negligible solubility of $\mathrm{TfOH}$ in many alcohols and ethers cannot be neglected, so we regard the organic solvent chemical nature as the third key point, because (together with its flowrate) it determines the ibuprofen recovery in the final crystallization, that should be preceded by a preconcentration.

\section{Acknowledgements}

The valuable help of the graduating students Giuseppe Grassi, MoS Industrial Chemistry, and Andrea Repetto, BS in Industrial chemistry, Università degli Studi di Milano, with the experimental tests and process modelling is gratefully acknowledged.

\section{List of Acronyms}

\begin{tabular}{ll}
\hline API & Active Pharmaceutical Ingredient \\
\hline AcOH & Acetic Acid \\
\hline HCI & Hydrochloric acid \\
\hline IBB & Isobutylbenzene \\
\hline KCI & Potassium chloride \\
\hline KOH & Potassium hydroxide \\
\hline
\end{tabular}




\begin{tabular}{ll}
\hline MeOH & Methanol \\
\hline NaCl & Sodium chloride \\
\hline NaOH & Sodium hydroxide \\
\hline NRTL & Non-Random Two Liquids \\
\hline PhI(OAC) 2 & Diacetoxyiodobenzene \\
\hline TfOH & Triflic acid, trifluoromethanesulfonic acid \\
\hline TfOK & Potassium trifluofomethansulfonate \\
\hline TMOF & Tetramethylorthoformate \\
\hline UNIFAC & Unified Activity Coefficients \\
\hline UNIQUAC & Universal Quasi-Chemical \\
\hline
\end{tabular}




\section{References}

[1] S.D. Schaber, D.I. Gerogiorgis, R. Ramachandran, J.M.B. Evans, P.I. Barton, B.L. Trout, Ind. Eng. Chem. Res. 2011, 50, 10083-10092 DOI: 10.1021/ie2006752.

[2] A. Sheldon, R., J. Chem. Biotechnol. 1997, 68, 381-388.

[3] N. Anderson, Practical Process Research and Development - A Guide for Organic Chemists, 2nd ed., Academic Press, oxford, 2012.

[4] M. Rouhi, A., Chem Eng News 2003, 81, 45-61 DOI: 10.1021/cen-v081n018.p045.

[5] I. Rossetti, M. Compagnoni, Chem. Eng. J. 2016, 296, 56-70 DOI: 10.1016/j.cej.2016.02.119.

[6] I. Rossetti, Catal. Today 2018, 308, 20-31.

[7] A.R. Bogdan, S.L. Poe, D.C. Kubis, S.J. Broadwater, D.T. McQuade, Angew. Chemie 2009, 121, 8699-8702 DOI: 10.1002/ange.200903055.

[8] H.G. Jolliffe, D.I. Gerogiorgis, Chem. Eng. Res. Des. 2015, 97, 175-191 DOI: 10.1016/j.cherd.2014.12.005.

[9] G. Zey, E., H. Shockley, T., A. Ryan, D., Method for Purification of Ibuprofen Comprising Mixtures, US5151551A, 1992.

[10] T. Lee, Y.W. Wang, Drug Dev. Ind. Pharm. 2009, 35, 555-567 DOI: 10.1080/03639040802459452.

[11] D.R. Snead, T.F. Jamison, Angew. Chemie 2015, 54, 983-987 DOI: 10.1002/anie.201409093.

[12] F. Villamil, O., Preferential Crystallization of a Racemic Compound via Its Conglomerate CoCrystals, DELFT University of Technology, 2016.

[13] H.G. Jolliffe, D.I. Gerogiorgis, Ind. Eng. Chem. Res. 2017, 56, 4357-4376 DOI: 10.1021/acs.iecr.6b02146.

[14] E. Goddard, L., A. Knesel, G., United States Patent, 5843863, 1998.

[15] A.C. Dimian, C.S. Bildea, A.A. Kiss, Integrated Design \& Simulation of Chemical Processes, II, Computer Aided Chemical Engineering, ELSEVIER, 2014. 
[16] AAVV, Aspen Plus User Guide, 10.2, Aspen Technology, Inc, 2000.

[17] R.L. Benoit, D. Figeys, Can. J. Chem. 1991, 69, 1985-1988 DOI: 10.1139/v91-286.

[18] A. Trummal, L. Lipping, I. Kaljurand, I.A. Koppel, I. Leito, J. Phys. Chem. A 2016, 120, 36633669 DOI: 10.1021/acs.jpca.6b02253.

[19] R.D. Howells, J.D. Mc Cown, Chem. Rev. 1977, 77, 69-92 DOI: 10.1021/cr60305a005.

[20] A.S. Teja, A.K. Gupta, K. Bullock, X.S. Chai, J. Zhu, Fluid Phase Equilib. 2001, 185, 265274 DOI: 10.1016/S0378-3812(01)00476-9.

[21] A. Kolker, J. De Pablo, Ind. Eng. Chem. Res. 1996, 35, 234-240 DOI: 10.1021/ie940621z.

[22] S.O. Yang, C.S. Lee, J. Chem. Eng. Data 1998, 43, 558-561 DOI: 10.1021/je970286w.

[23] K. Alfonsi, J. Colberg, P.J. Dunn, T. Fevig, S. Jennings, T.A. Johnson, H.P. Kleine, C. Knight, M.A. Nagy, D.A. Perry, M. Stefaniak, Green Chem. 2008, 10, 31-36 DOI: 10.1039/B711717E.

[24] I. Rodríguez Donis, V. Gerbaud, X. Joulia, Ind. Eng. Chem. Res. 2016, 40, 4935-4950 DOI: 10.1021/ie0010363.

[25] C.A. Shukla, A.A. Kulkarni, Beilstein J. Org. Chem. 2017, 13, 960-987 DOI: 10.3762/bjoc.13.97.

[26] A. Skrzecz, D.G. Shaw, A. Maczynski, J. Phys. Chem. Ref. Data 1999, 28, 983-1235 DOI: 10.1063/1.556052.

[27] E. Gordon, R., A. Sanjay, I., Crystallization of Ibuprofen, US Pat. 4476248, 1983.

[28] M.A. Filippa, E.I. Gasull, Fluid Phase Equilib. 2013, 354, 185-190 DOI: 10.1016/j.fluid.2013.06.032.

[29] S. Gracin, A.C. Rasmuson, J. Chem. Eng. Data 2002, 47, 1379-1383 DOI: 10.1021/je0255170.

[30] L.C. Garzón, F. Martínez, J. Solution Chem. 2004, 33, 1379-1395 DOI: 10.1007/s10953-0041051-2.

[31] I. Hahnenkamp, G. Graubner, J. Gmehling, Int. J. Pharm. 2010, 388, 73-81 DOI: 
10.1016/j.ijpharm.2009.12.036.

[32] H. Bagheri, S. Ghader, N. Hatami, Chem Technol Ind J 2017, 12, 1-14.

[33] A. Rashid, E.T. White, T. Howes, J.D. Litster, I. Marziano, Chem. Eng. Res. Des. 2012, 90, 158-161 DOI: 10.1016/j.cherd.2011.08.003. 


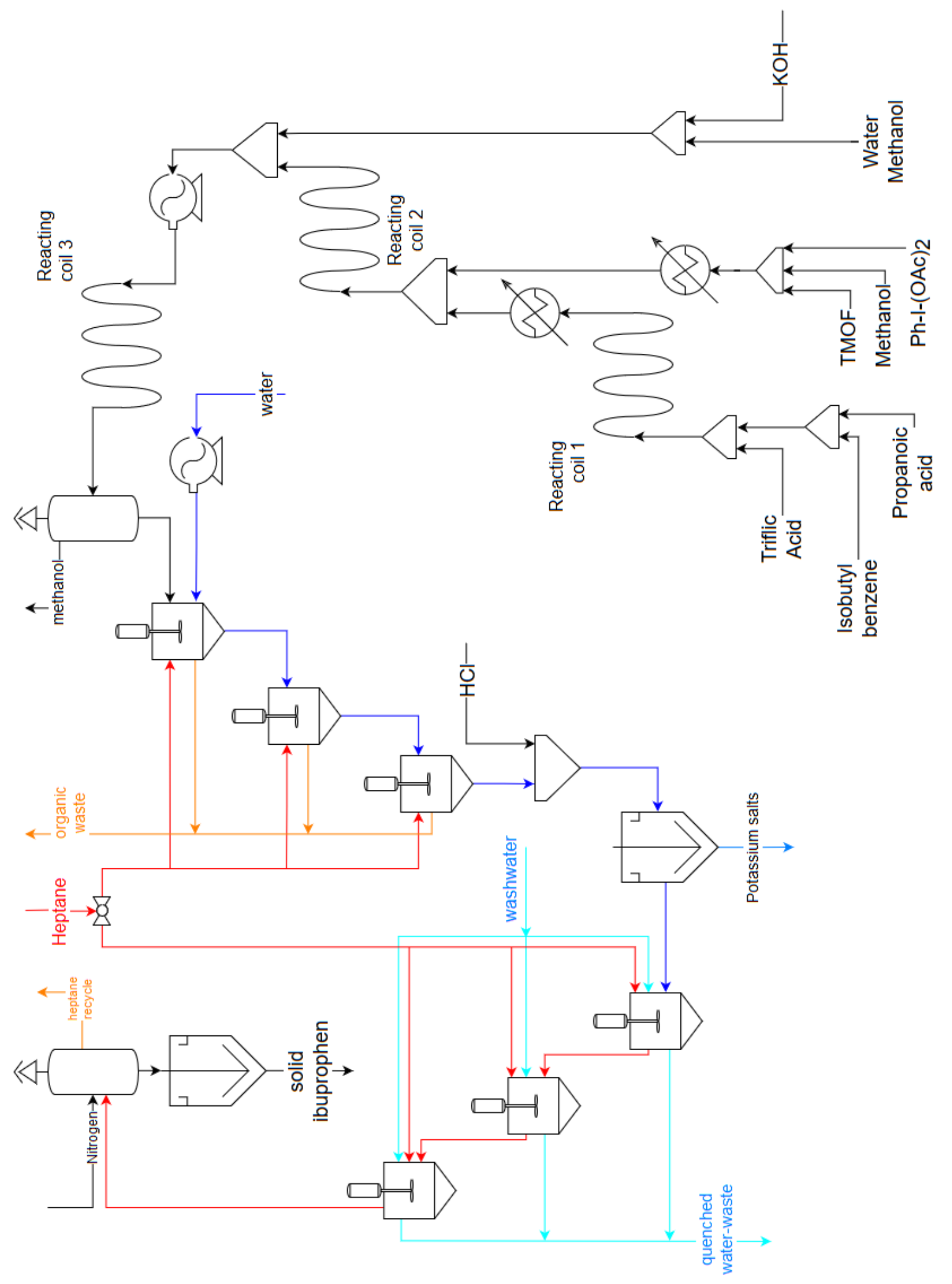

Figure 1: Process flowsheet. 


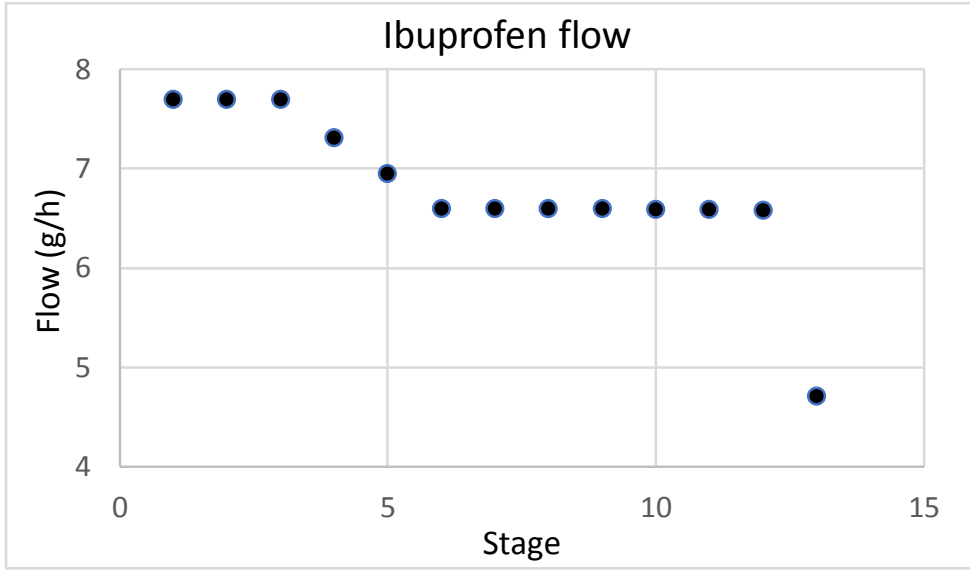

Figure 2: Mass flow of ibuprofen through the main separation stages. 


\section{Table of Contents}

The continuous flow manufacturing of ibuprofen was studied to highlight the criticisms during process design for API synthesis. The correct selection of the thermodynamic model to represent unconventional reactants, products and intermediates revealed compulsory to proper account for heat release/supply and for phase separations. Appropriate computation of electrolytes is also critical. 